\title{
Effect of angioembolisation versus surgical packing on mortality in traumatic pelvic haemorrhage: $A$ systematic review and meta-analysis
}

\author{
Ahmed El Muntasar ${ }^{1}$, Ethan Toner ${ }^{1}$, Oddai A. Alkhazaaleh ${ }^{1}$, Danaradja Arumugam ${ }^{1}$, Nikhil Shah ${ }^{2}$, Shahab \\ Hajibandeh $^{3}$, Shahin Hajibandeh ${ }^{4}$ \\ ${ }^{1}$ Department of General Surgery, Royal Blackburn Hospital, Blackburn, UK \\ ${ }^{2}$ Department of Trauma and Orthopaedics, Wrightington, Wigan and Leigh NHS Foundation Trust, UK \\ ${ }^{3}$ Department of General Surgery, Salford Royal Hospital, Salford, UK \\ ${ }^{4}$ Department of General Surgery, North Manchester General Hospital, Manchester, UK
}

Corresponding Author: Shahin Hajibandeh,Email: shahin_hajibandeh@yahoo.com

BACKGROUND: The management of complex pattern of bleeding associated with pelvic trauma remains a big challenge for trauma surgeons. We aimed to conduct a comprehensive meta-analysis to compare the outcomes of angioembolisation and pelvic packing in patients with pelvic trauma.

METHODS: We conducted a systematic search of electronic information sources, including MEDLINE; EMBASE; CINAHL; the CENTRAL; the World Health Organization International Clinical Trials Registry; ClinicalTrials.gov; ISRCTN Register, and bibliographic reference lists. The primary outcome was defined as mortality. Combined overall effect sizes were calculated using randomeffects models. Results are reported as the odds ratio $(O R)$ and $95 \%$ confidence interval $(C l)$.

RESULTS: We identified 3 observational studies reporting a total of 120 patients undergoing angioembolisation $(n=60)$ or pelvic packing $(n=60)$ for pelvic trauma. Reporting of the Injury Severity Score (ISS) was variable, with higher ISS in the pelvic packing group. The risk of bias was low in two studies, and moderate in one. The pooled analysis demonstrated that angioembolisation did not significantly reduce mortality in patients with pelvic trauma compared to surgery $(\mathrm{OR}=1.99 ; 95 \% \mathrm{Cl}=$ $0.83-4.78, P=0.12)$. There was mild between-study heterogeneity $\left(I^{2}=0 \%, P=0.65\right)$.

CONCLUSION: Our analysis found no significant difference in mortality between angioembolisation and pelvic packing in patients with traumatic pelvic haemorrhage. The current level of evidence in this context is very limited and insufficient to support the superiority of a treatment modality. Future research is required.

KEY WORDS: Pelvic trauma; Pelvic packing; Angiography; Embolisation

World J Emerg Med 2018;9(2):85-92

DOI: 10.5847/wjem.j.1920-8642.2018.02.001

\section{INTRODUCTION}

The management of pelvic fractures with associated blunt abdominal trauma remains a challenging topic for trauma surgeons. ${ }^{[1]}$ The difficulty arises from the complex pattern of bleeding, resulting in a mortality as high as $40 \%$. Furthermore, rapid recognition and management of the haemorrhage is crucial, particularly in the first 24 hours, as the mortality increases to $60 \%$ due to uncontrolled haemorrhage and multiple blood transfusions leading to multi-organ failure. ${ }^{[2,3]}$

High-energy impact to the pelvis is associated with high mortality due to disturbance in the pelvic ring and transfer of the high energy to the rest of the body. ${ }^{[4]} \mathrm{Up}$ to $90 \%$ of patients with pelvic trauma have injuries in other parts of the body with $64 \%$ being intra-abdominal. ${ }^{[4]}$ Haemodynamic instability, co-morbidities, time constraints and unclear mechanisms of injury make the decision making regarding the most appropriate management strategy in patients with 
abdominopelvic trauma very challenging. ${ }^{[5,6]}$ The source of bleeding in pelvic fractures and blunt abdominal trauma is often multifactorial and unclear in origin, with most patients having both pelvic and intraperitoneal bleeding. ${ }^{[6,7]}$

Angiography and embolisation, first discussed in the literature in 1972 , are quoted to be successful in managing $80 \%-100 \%$ of arterial bleeds. However, arterial bleeds account for only $10 \%$ of pelvic fracture haemorrhages. ${ }^{[1,8]}$ The mortality following a successful angioembolisation has been reported to be as high as $50 \%{ }^{[1,4]}$ Interestingly, postmortem and clinical studies have demonstrated that the cause of death in pelvic injury is due to haemorrhage without major arterial injury. ${ }^{[1,9]}$

Pelvic packing for pelvic injury was first carried out in the 1960s. Using the transperitoneal approach, it was initially associated with high mortality due to incision of the intact peritoneum and disruption of the pelvic haematoma. ${ }^{[1]}$ The packing technique was innovated later as packing was performed in the retroperitoneal space leaving the peritoneum intact. Retroperitoneal pelvic packing is quicker, associated with minimal intraoperative blood loss and avoids unnecessary angiography. ${ }^{[1]}$ It is most commonly used to manage venous bleeds, which account for more than $80 \%$ of pelvic haemorrhage. ${ }^{[6,10]}$ Tamponade for haemostasis of pelvic haemorrhage has been shown to be beneficial when applied as part of the treatment algorithm, including rapid resuscitation, early stablisation and immediate surgical revision of the pelvis. ${ }^{[1]]}$

To our knowledge there is no meta-analysis that explored the literature regarding the management of haemorrhage due to pelvic trauma. We aimed to conduct a comprehensive systematic review and meta-analysis to compare the outcomes of angioembolisation and pelvic packing in patients with pelvic trauma.

\section{METHODS}

\section{Design and study selection}

We pre-specified the inclusion criteria, methods, and outcomes of our study in a review protocol. The study was conducted according to the Preferred Reporting Items for Systematic Reviews and Meta-Analyses (PRISMA) statement standards. ${ }^{[12]}$

We planned to select randomised controlled trials (RCTs) and observational studies comparing the outcomes of angioembolisation and pelvic packing in patients with pelvic trauma induced haemorrhage.
Patients of any age and gender with pelvic trauma who had a CT confirmed pelvic arterial or venous injury, persistently low haemobglobin or with unstable haemodynamic status were considered for inclusion. The intervention of interest was angioembolisation. The primary intervention was compared with pelvic packing.

Primary outcome was defined as perioperative mortality. Perioperative complications, procedure time, length of hospital stay were the secondary outcomes.

\section{Literature search strategy}

Two authors (AEM and ET) independently searched the following electronic databases: MEDLINE, EMBASE, CINAHL, and the Cochrane Central Register of Controlled Trials (CENTRAL). The last search was run on 16 May 2017. The search strategy, is presented in Table 1. Moreover, we searched the reference lists of relevant articles and reviews for further relevant articles.

\section{Selection of studies}

Two authors (AEM and ET) independently assessed the title and abstract of articles identified through literature searches. The full-texts of relevant reports were assessed and those articles that met the inclusion criteria of our study were selected. We resolved discrepancies in study selection by discussion between the authors. An independent third author (SH) was consulted in the event of disagreement.

\section{Data extraction and management}

We created an electronic data extraction spreadsheet in line with the Cochrane's data collection form for intervention reviews. We pilot-tested the spreadsheet in randomly selected articles and adjusted it accordingly. Our data extraction spreadsheet included the following information: (1) study-related data (first author, year of publication, country of origin of the corresponding author, journal in which the study was published, study

\begin{tabular}{ll}
\multicolumn{2}{l}{ Table 1. Search strategy } \\
\hline Search no. & Search strategy \\
\hline$\# 1$ & Pelvic injury \\
$\# 2$ & Abdominopelvic trauma \\
$\# 3$ & Pelvic trauma \\
$\# 4$ & $\# 1$ OR \#2 OR \#3 \\
$\# 5$ & Pelvic packing \\
$\# 6$ & Surgical packing \\
$\# 7$ & Surgery \\
$\# 8$ & Trauma surgery \\
$\# 9$ & \#5 or \#6 or \#7 or \#8 \\
$\# 10$ & Embolisation \\
$\# 11$ & Embolization \\
$\# 12$ & Angio \\
$\# 13$ & \#10 OR \#11 OR \#12 \\
$\# 14$ & $\# 4$ AND \#9 AND \#13 \\
\hline
\end{tabular}


design, study size, and type of intervention); (2) baseline demographic and clinical information of the study populations [age, the injury severity score (ISS), and fracture type]; (3) primary and secondary outcome data.

Two authors (AEM and ET) independently collected and recorded data in the data extraction spreadsheet. Disagreements were resolved by discussion. If no agreement could be reached, a third author $(\mathrm{SH})$ was consulted.

\section{Assessment of risk of bias}

The methodological quality and risk of bias of the included articles were assessed independently by two authors (AEM and ET). We used the Newcastle-Ottawa scale $(\mathrm{NOS})^{[13]}$ observational studies, respectively. The NOS uses a star system with a maximum of nine stars to evaluate a study in three domains: the selection of the study groups, the comparability of the groups, and the ascertainment of outcome of interest. We judged studies that received a score of nine stars to be at low risk of bias, studies that scored seven or eight stars to be at medium risk, and those that scored six or less to be at high risk of bias. We resolved disagreements by discussion between the two assessing authors. If no agreement could be reached, a third reviewer ( $\mathrm{SH}$ ) acted as an adjudicator.

\section{Summary measures and synthesis}

Our primary outcome (mortality) was a dichotomous outcome; therefore, we calculated the odds ratio $(O R)$ as the summary measures. The $O R$ is the odds of an adverse event in the angioembolisation group compared to the pelvic packing group. An $O R$ of less than one would favour the angioembolisation group and an $O R$ of more than one would favour the pelvic packing group.

We used the individual patient as the unit of analysis. We recorded information about dropouts, withdrawals and other missing data and, if not reported, we contacted the study authors. The final analysis was based on intention-to-treat data from the individual clinical studies where possible.

We used the Review Manager 5.3 software for data synthesis. ${ }^{[12]}$ The extracted data were entered into Review Manager by the first independent author ( $\mathrm{SH}$ ) and checked by a second independent author (SH). We used random-effects or fixed-effect modelling, as appropriate, for analysis. We applied random-effects models if considerable heterogeneity among the studies, as defined by Higgins et al ${ }^{[12]}$ was identified. The results were reported in a forest plot with $95 \%$ confidence intervals (CIs).

Heterogeneity among the studies was assessed using the Cochran Q test $\left(\chi^{2}\right)$. We quantified inconsistency by calculating $I^{2}$ and interpreted it using the following guide: $0 \%$ to $25 \%$ might not be important; $25 \%$ to $75 \%$ : may represent moderate heterogeneity; $75 \%$ to $100 \%$ may represent substantial heterogeneity. We planned to use the Comprehensive Meta-Analysis (CMA) software (Biostat, Englewood, NJ) to calculate the Egger's regression intercept to formally assess reporting bias in our review. Also, we planned to construct funnel plots and evaluate their symmetry to visually assess publication bias, as long as a sufficient number of studies (more than 10) were available.

Additional analyses were planned to explore potential sources of heterogeneity and assess the robustness of our results. For each outcome, we repeated the primary analysis using random-effects or fixed-effect models. In addition, we calculated the pooled $O R$, risk ratio $(R R)$, or risk difference $(R D)$ for each dichotomous variable. We assessed the effect of each study on the overall effect size and heterogeneity by repeating the analysis after removing one study at a time

\section{RESULTS}

The searches identified 874 articles. Following reviewing the titles and abstracts, which was followed by assessing the full text, 3 articles ${ }^{[14-16]}$ were identified, all retrospective cohort studies (Figure 1).

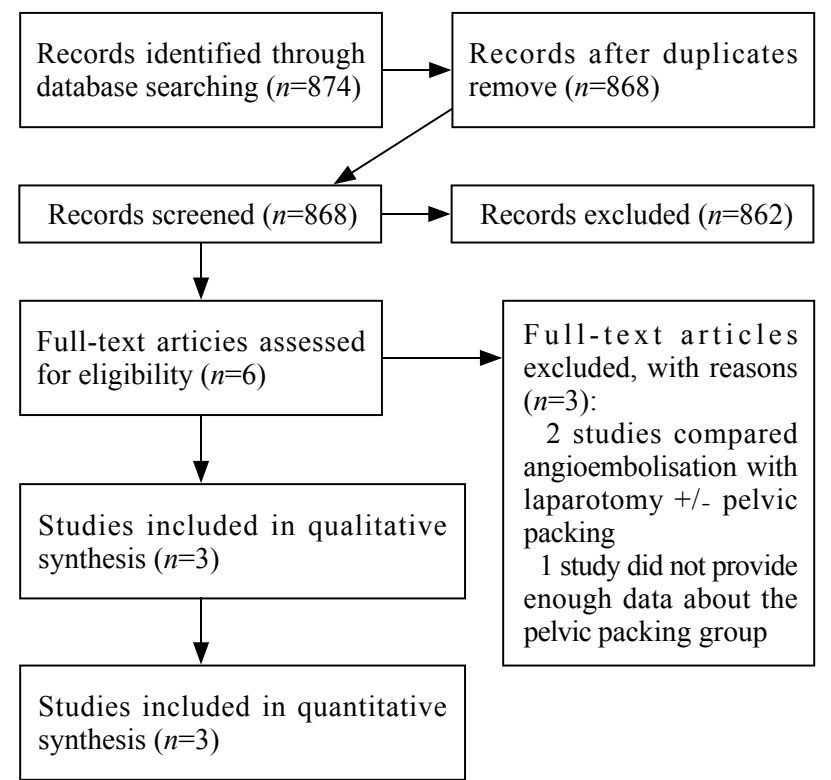

Figure 1. Study flow diagram. 
The included studies reported a total of 120 patients, of whom 60 underwent angioembolisation and the remaining 120 underwent pelvic packing. The majority of the included patients were males. The patients in angioembolisation and pelvic packing groups in each study were of comparable mean age; however, there were differences in mean age between the included studies. The ISS were similar between the treatments groups in one of the included studies; however, in two studies the ISS was significantly higher in the pelvic packing groups. The study-related data are presented in Table 2. Baseline demographic and clinical characteristics of the study populations are outlined in Table 3 . All of the included studies classified the pelvic fractures and stated the type and rate of associated injuries. Two studies used the Young and Burgess classification, and one used Tile's classification. Most of the included patients had major pelvic fracture. However, differences in fracture type distribution were not statistically significant.

\section{Assessment of bias}

Table 4 presents the risk of bias assessment of the included studies. The risk of bias were low in 2 studies and moderate in one.

\section{Included studies}

Tai et al ${ }^{[14]}$ conducted a retrospective review at a single trauma centre. They compared the outcomes of early pelvic angiography and retroperitoneal pelvic packing in the management of haemodynamically unstable pelvic fractures over a 2 year period. The study had 2 arms, angioembolisation group $(n=13)$ and pelvic packing group $(n=11)$. Moreover, patients in their pelvic packing group who developed persistent hemorrhage (persistent hypotension and need for blood transfusion) after pelvic packing $(n=5)$ underwent pelvic angiography. Mortality, blood products transfusion, time spent in the accident and emergency department and systolic blood pressure were the measured outcomes. Their main finding was that early pelvic packing followed by angiography had a similar outcome to angiography with embolisation in the management of haemodynamically unstable patients post pelvic fracture.

Osborn et a ${ }^{[15]}$ conducted a retrospective cohort study which evaluated the outcomes of pelvic packing and angiographic embolisation in haemodynamically

Table 2. Characteristics of included studies

\begin{tabular}{|c|c|c|c|c|}
\hline Author & Year & Country & Journal & Type of study \\
\hline Tai et al $^{[14]}$ & 2011 & China & J Trauma Inj Infect Crit Care & Retrospective observational study \\
\hline Osborn et $\mathrm{al}^{[15]}$ & 2009 & USA & Injury & Retrospective observational study \\
\hline Li et $\mathrm{al}^{[16]}$ & 2016 & China & Injury & Retrospective observational study \\
\hline
\end{tabular}

Table 3. Baseline demographic and clinical characteristics

\begin{tabular}{|c|c|c|c|c|c|c|c|c|}
\hline Author & Number of patients & $\mathrm{AE}$ & Packing & Mean age $^{a}$ & Haemodynamic status & $\mathrm{ISS}^{\mathrm{a}}$ & Major fracture $^{b}$ & Minor fracture $^{c}$ \\
\hline Tai et al ${ }^{[14]}$ & 24 & 13 & 11 & $\begin{array}{c}44.8 \pm 24.7 \\
\text { vs. } \\
51.2 \pm 19 \\
P=0.490\end{array}$ & Unstable & $\begin{array}{c}42.3 \pm 18.8 \\
\text { vs. } \\
40 \pm 12.5 \\
P=0.732\end{array}$ & $\begin{array}{c}72.7 \% \\
\text { VS. } \\
53.9 \%\end{array}$ & $\begin{array}{c}27.3 \% \\
\text { VS. } \\
46.1 \%\end{array}$ \\
\hline Osborn et $\mathrm{al}^{[15]}$ & 40 & 20 & 20 & $\begin{array}{c}39.5 \pm 17.4 \\
\text { vs. } \\
37.9 \pm 18.9 \\
P=\mathrm{NS}\end{array}$ & Unstable & $\begin{array}{c}45.9 \pm 8.7 \\
\text { Vs. } \\
54.7 \pm 12.7 \\
P=0.014\end{array}$ & $\begin{array}{c}70.0 \% \\
\text { VS. } \\
65.0 \%\end{array}$ & $\begin{array}{c}30.0 \% \\
\text { VS. } \\
35.0 \%\end{array}$ \\
\hline Li et $\mathrm{al}^{[16]}$ & 56 & 27 & 29 & $\begin{array}{c}43 \pm 13 \text { vs. } 40 \pm 9 \\
P=0.373\end{array}$ & Unstable & $\begin{array}{c}48 \pm 6 \text { vs. } 43 \pm 7 \\
P=0.005\end{array}$ & All patients & NA \\
\hline
\end{tabular}

AE: angioembolisation; ISS: Injury Severity Socre; NS: non-significant; NA: not applicable; a: angioembolisation versus laparotomy; b: major fractures included lateral compression type III, anteroposterior compression types II and III, vertical shear fracture types I, II, and III, and combined mechanism fractures; c: minor fractures included lateral compression types I, II, and anteroposterior compression fractures type I.

Table 4. Methodological quality of the observational studies assessed with the Newcastle-Ottawa Scale ${ }^{[13]}$

\begin{tabular}{|c|c|c|c|c|c|c|c|c|c|}
\hline Author & $\begin{array}{l}\text { Representativeness } \\
\text { of the exposed } \\
\text { cohort }\end{array}$ & $\begin{array}{l}\text { Selection } \\
\text { of the non- } \\
\text { exposed } \\
\text { cohort }\end{array}$ & $\begin{array}{l}\text { Ascertainment } \\
\text { of exposure }\end{array}$ & $\begin{array}{l}\text { Demonstration } \\
\text { that outcome of } \\
\text { interest was not } \\
\text { present at start of } \\
\text { study }\end{array}$ & $\begin{array}{l}\text { Comparability } \\
\text { of cohorts on the } \\
\text { basis of the design } \\
\text { or analysis }\end{array}$ & $\begin{array}{l}\text { Assessment } \\
\text { of outcome }\end{array}$ & $\begin{array}{l}\text { Was follow-up } \\
\text { long enough } \\
\text { for outcomes } \\
\text { to occur }\end{array}$ & $\begin{array}{l}\text { Adequacy } \\
\text { of follow } \\
\text { up of } \\
\text { cohorts }\end{array}$ & $\begin{array}{l}\text { Total } \\
\text { score }\end{array}$ \\
\hline Tai et al ${ }^{[14]}$ & $*$ & $*$ & $*$ & * & * & $*$ & $*$ & * & 9 \\
\hline $\begin{array}{l}\text { Osborn et } \\
\mathrm{al}^{[15]}\end{array}$ & $*$ & $*$ & $*$ & $*$ & $*$ & $*$ & * & $*$ & 8 \\
\hline Li et $\mathrm{al}^{[16]}$ & * & $*$ & * & * & * & * & * & * & 9 \\
\hline
\end{tabular}


unstable patients with pelvic trauma in a level I trauma centre. The study included an equal cohorts of 20 patients for each intervention. The data was collected over an 8 year period. The study had two arms, the first was the cohort which received urgent operative sheet removal, placement of external fixation and pelvic packing. The second cohort, was treated with emergency endovascular embolisation. Mortality, systolic blood pressure, blood lactate level, length of intensive care unit (ICU) stay and length of hospital stay were the outcome parameters. They concluded that both techniques; pelvic packing and angiography, carried similar outcomes in managing haemodynamically unstable pelvic fracture patients.

Li et $\mathrm{al}^{[16]}$ conduced a retrospective cohort study which investigated the outcomes of pelvic packing and angioembolisation in patients with haemodynamically unstable pelvic fractures. The study included 2 arms, angioembolisation group $(n=27)$ and pelvic packing group $(n=29)$. Primary outcome of their study was mortality. Moreover, post-procedural complications, time from admission to angiography/ surgery, procedure time, length of ICU, postoperative blood transfusion units administered, and secondary procedures were the secondary outcome parameters. There was no significant difference in mortality rate between two groups ( $P=0.449)$. The study authors concluded that compared with angioembolisation, pelvic packing has shorter time to intervention and surgical time. ${ }^{[15]}$ Therefore, pelvic packing was recommended as the more rapid treatment of severe pelvic trauma than pelvic angioembolisation. Furthermore, the authors recommended that pelvic packing is suitable for patients with haemodynamic instability at centers where the interventional radiology staff is not in-house at all times.

\section{Excluded studies}

Hauschild et $\mathrm{al}^{[17]}$ conducted a multi-centre (23 trauma centres) prospective cohort study which included 152 patients with pelvic fractures and associated vascular injuries, either arterial or venous injuries. Data was collected using the German pelvic trauma register. The study had 2 arms of intervention. One group (noembolisation group) received conventional methods of haemorrhage control such as external stabilisation and preperitoneal packing and the other group (embolization group) underwent angioembolisation. Any patient who had a CT confirmed pelvic vascular injury, persistently low haembglobin or was haemodynamically unstable was eligible for angioembolisation. Mortality, complications, number of blood transfusions and length of hospital stay were measured as outcomes for the study. The study findings showed that angioembolisation increased the rate of blood transfusions, thus leading to more episodes of adult respiratory distress syndrome and multi-organ failure. Despite this, there was no significant difference in the mortality rate between two groups. The study concluded that angioembolisation should be utilized with and supplement conventional measures of pelvic haemorrhage control. This study was excluded as no data regarding the pelvic packing group was provided.

Katsura et a ${ }^{[18]}$ conducted a multi-centre retrospective cohort study, which included 317 patients suffering from pelvic fracture and haemoperitoneum. The study had 2 arms of intervention; laparotomy first versus angiographic embolisation first. In hospital mortality and mortality within 24 hours of admission were the primary and secondary outcome measures, respectively. The study found no significant difference in mortality between the laparotomy and angioembolisation groups. The authors concluded that initial intervention using either laparotomy or angiographic embolisation did not lead to an increased risk of mortality, and either proved a suitable choice of intervention. This study was also excluded as no data regarding the pelvic packing was provided.

Fang et al ${ }^{[19]}$ conducted a single-centre cohort study from a level 1 trauma center which included 545 patients with pelvic fractures and associated arterial haemorrhage. Data was collected over a 10 -year period. In this study, 43 patients underwent angioembolisation and 33 patients underwent exploratory laparotomy. The study found no difference in mortality rates between the two groups; however, patients undergoing angioembolisation had higher rates of blood transfusion. This study was also excluded as no data regarding the pelvic packing was provided.

\section{Outcome}

Outcomes are summarised in Figure 2.

All included studies evaluated mortality as an outcome. There were $20(33.3 \%)$ deaths in the angioembolisation group whereas $12(20.0 \%)$ patients died in the pelvic packing group. The pooled analysis of 120 patients did not find any significant difference in mortality between angioembolisation and pelvic packing groups despite the existence of a trend in favour of the later $(O R=1.99 ; 95 \% C I=0.83-4.78, P=0.12)$. Betweenstudy heterogeneity was low $\left(I^{2}=0 \%, P=0.65\right)$. 
The included studies did not provide appropriate data for analysis of perioperative complications, procedure time, and length of hospital stay,

\section{Sensitivity analysis}

Using random-effects or fixed-effect models did not affect the pooled effect size in any of the outcomes. The direction of pooled effect size remained unchanged when the OR, RR, or RD was calculated. Removal of one study at a time did not change the direction of pooled effect size in favour of either intervention.

\section{DISCUSSION}

To our knowledge there has been no meta-analysis comparing the outcomes of endovascular embolisation and pelvic packing in patients with haemodynamically unstable pelvic trauma. Therefore, we conducted a comprehensive systemic review of the literature and meta-analysis of the related outcomes and identified 3 observational studies ${ }^{[13-15]}$ reporting a total of 120 patients with traumatic pelvic haemorrhage of whom 60 underwent angioembolisation and the remaining 60 underwent surgical pelvic packing. Our analysis demonstrated that there was no significant difference in mortality between angioembolisation and pelvic packing in the management of traumatic pelvic haemorrhage. The heterogeneity among the included studies was low indicating that the outcome of our analysis may be robust.

The rationale of pelvic packing is haemostasis by direct compression of venous bleeding from the presacral plexus or cancellous bone fracture surfaces. ${ }^{[1,16]}$ During the procedure even obvious proximal arterial haemorrhage in the retroperitoneum can be controlled. ${ }^{[15]}$ Pelvic packing has been reported to be associated with inpatient mortality of $20 \%-36 \%{ }^{[14-16,20,21]}$ Coccolini et al ${ }^{[22]}$ recently published the World Society of Emergency Surgery (WSES) classification of pelvic trauma and the management Guidelines. They recommended that patients with pelvic fracture-related hemodynamic instability should always be considered for preperitoneal pelvic packing, especially in hospitals with no angiography service. ${ }^{[22]}$ Moreover, they suggested that direct preperitoneal pelvic packing represents an effective surgical measure of early haemorrhage control in hypotensive patients with bleeding pelvic ring disruptions. ${ }^{[22]}$ Furthermore, they highlighted that pelvic packing should be performed in conjunction with pelvic stabilization to maximize the effectiveness of bleeding control. ${ }^{[22]}$ Finally, the authors recommended that patients with pelvic fracture-related hemodynamic instability with persistent bleeding after angiography should always be considered for pre-peritoneal pelvic packing. ${ }^{[22]}$

The studies of Hauschild et al, ${ }^{[17]}$ Tai et al, ${ }^{[14]}$ and Osborn et $\mathrm{al}^{[15]}$ found that surgical packing reduced the requirement for blood transfusion compared with angioembolisation. Repeated blood transfusions can lead to Acute Respiratory Distress Syndrome (ARDS) as well as contributing to multi-organ failure ${ }^{[23-25]}$ Hauschild et $\mathrm{al}^{[17]}$ found more ARDS and multi-organ failure in their angioembolisation group although this was not statistically significant.

Angioembolisation is reported to be successful in management of $80 \%-100 \%$ of arterial bleeds. However, arterial bleeds only account for $10 \%$ of pelvic fracture haemorrhages. ${ }^{[1,9]}$ Therefore, angioembolisation is expected to benefit only a small number of patients with pelvic haemorrhage. Miller et $\mathrm{al}^{[25]}$ reported $26 \%$ and Osborn et al ${ }^{[15]}$ reported $30 \%$ mortality following angioembolisation for pelvic haemorrhage. However, Tai et al ${ }^{[14]}$ reported more than $69 \%$ mortality associated with primary angioembolisation. In all of the three aforementioned studies $33 \%$ died due to massive

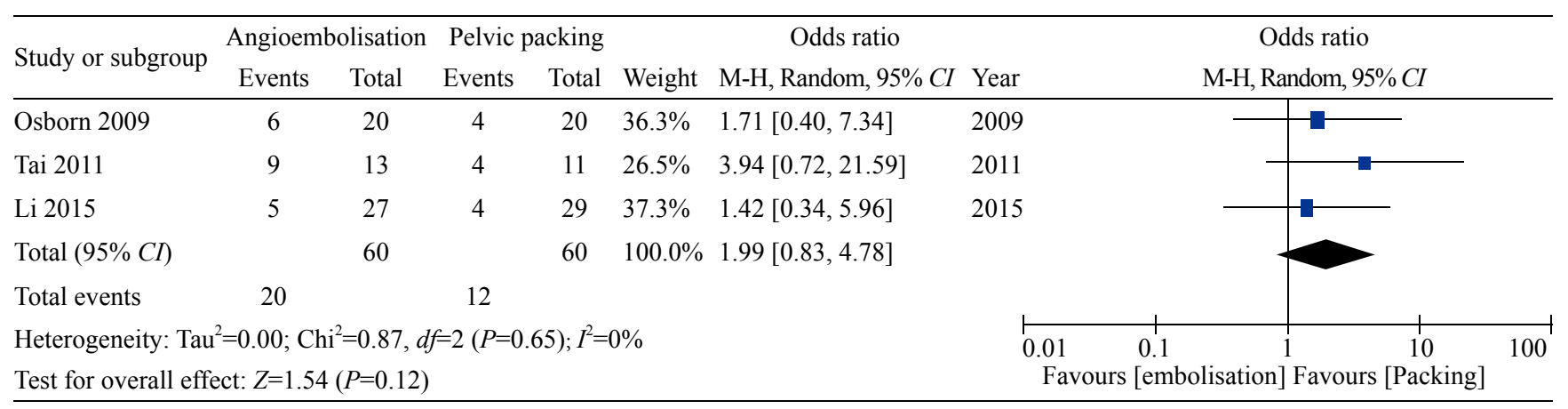

Figure 2. Forest plots of comparison of mortality. The solid squares denote the odds ratios (ORs). The horizontal lines represent the $95 \%$ confidence intervals (CIs), and the diamond denotes the pooled effect size. M-H, Mantel Haenszel test. 
haemorrhage in the angioembolisation group..$^{[14,15,26]}$ Miller et $\mathrm{al}^{[25]}$ found that angioembolisation should be used once extra-pelvic and non-arterial sources of bleeding are controlled. Furthermore, Coccolini et $\mathrm{al}^{[22]}$ recommended that following pelvic stabilization, aggressive resuscitation and exclusion of extra-pelvic haemorrhage, patients with pelvic fractures and unstable hemodynamic status or evidence of ongoing bleeding should be considered for pelvic angioembolisation.

Angioembolisation is a time-consuming procedure and it involves transportation of a severely injured patient to an angiography suite. ${ }^{[14,16]}$ It requires the availability of specialised instruments and trained interventional radiologists. However, in the majority of centers, interventional radiologist is not in house at all times. ${ }^{[27]}$ Some of the included studies considered time to intervention using either early angioembolisation or early pelvic packing. Tai et $\mathrm{al}^{[14]}$ and Osborn et $\mathrm{al}^{[15]}$ found that angioembolisation took longer to implement. This can be argued that time taken for implementing angioembolisation can be utilized for immediate surgical packing. Hauschild et a ${ }^{[17]}$ suggested that angioembolisation should be used to supplement conventional measures such as packing or external fixation, as they found a non-significant reduction in mortality when angioembolisation was used in combination with conventional measures. Surgical packing can be used to gain rapid control of haemorrhage in the pelvic cavity by impeding venous bleeding. ${ }^{[1]}$ This could be a useful initial stabilization method to attempt to control haemorrhage prior to deciding to perform angioembolisation if required for further control of arterial bleeding.

Our study has some limitations. No RCT, the gold standard study design for comparative studies, was identified to provide high quality evidence for or against angioembolisation or pelvic packing. The available evidence comes mainly from a limited number of heterogeneous retrospective cohort studies with small sample sizes that are inevitably subject to selection bias. In the study of Tai et al, ${ }^{[14]} 5$ out of 11 patients in the pelvic packing group developed persistent heamorrhage following the procedure and received subsequent pelvic angiography. This undoubtedly subjected the outcome of that study, and subsequently our study, to a major bias as the potential therapeutic advantages of angiography in those patients have been credited to pelvic packing. The included studies did not provide appropriate data for analysis of any of the defined secondary outcome parameters. Moreover, the ISS was significantly higher in the pelvic packing groups in 2 of the included studies. Considering the fact that ISS, not type of pelvic instability, appears to be the most important factor in predicting mortality in patients with pelvic fractures, ${ }^{[1]}$ higher ISS in the pelvic packing groups might have affected the outcomes in favour of angioembolisation in those studies.

\section{CONCLUSIONS}

Our analysis of best available evidence from heterogeneous observational studies with small sample sizes found no significant difference in mortality between angioembolisation and pelvic packing in patients with traumatic pelvic haemorrhage. Despite the fact that management of pelvic trauma has been intensively discussed in the literature in recent years, the current level of evidence in this context is very limited and insufficient to support the superiority of a treatment modality. Future high level research is required to understand the role of each treatment modality in the algorithm of managing a pelvic ring injury and associated haemodynamic instability. However, the nature of pelvic trauma, particularly in haemodynamically unstable patients, will be a big challenge to design such a research.

Funding: This systematic review did not receive any funding. Ethical approval: Not needed.

Conflicts of interest: The authors declare that they have no competing interests.

Contributors: conception and design: $\mathrm{SH}, \mathrm{SH}$; literature search and study selection: AEM, ET, SH; data collection: AEM, ET, SH; analysis and interpretation: $\mathrm{SH}, \mathrm{SH}$; Writing the article: AEM, ET, $\mathrm{SH}, \mathrm{SH}$; critical revision of the article: AEM. ET, OAA, DA, NS, $\mathrm{SH}, \mathrm{SH}$; final approval of the article: AEM. ET, OAA, DA, NS, $\mathrm{SH}, \mathrm{SH}$; statistical analysis: $\mathrm{SH}, \mathrm{SH}$; overall responsibility of the project: $\mathrm{SH}, \mathrm{SH}$.

\section{REFERENCES}

1 White C, Hsu J, Holcomb J. Haemodynamically unstable pelvic fractures. Injury. 2009; 40(10):1023-30.

2 Suzuki T, Smith WR, Moore EE. Pelvic packing or angiography: competitive or complementary? Injury. 2009;40(4):343-53.

3 Grotz MR, Gummerson NW, Gänsslen A, Petrowsky H, Keel M, Allami MK, et al. Staged management and outcome of combined pelvic and liver trauma. An international experience of the deadly duo. Injury. 2006;37(7): 642-51.

4 Heetveld M, Harris I, Schlaphoff G, Sugrue M. Guidelines for the management of haemodynamically unstable pelvic fracture 
patients. ANZ J Surg. 2002;74(7):520-9.

5 Agolini S, Shah K, Jaffe J, Newcomb J, Rhodes M, Reed $\mathrm{J}$. Arterial embolization is a rapid and effective technique for controlling pelvic fracture hemorrhage.J Trauma. 1997;43(3):395-9.

6 Spahn D, Cerny V, Coats T, Duranteau J, Fernández-Mondéjar E, Gordini G, et al. Management of bleeding following major trauma: a European guideline. Crit Care. 2007;11(1):R17.

7 Starr AJ. Arterial embolization is a rapid and effective technique for controlling pelvic fracture hemorrhage. J Trauma. 1998;44(1):236.

8 Papakostidis C, Giannoudis P. Pelvic ring injuries with haemodynamic instability: efficacy of pelvic packing, a systematic review. Injury. 2009;40 Suppl 4:S53-61.

9 Huittinen VM, Slatis P. Postmortem angiography and dissection of the hypogastric artery in pelvic fractures. Surgery. 1973;73(3):454-62.

10 Agolini SF, Kamalesh S, Jaffe J, Newcomb J, Rhodes M, Reed JF 3rd. Arterial embolization is a rapid and effective technique for controlling pelvic fracture hemorrhage. J Trauma. 1997;43(3):395-9.

11 Pohlemann T, Gansslen A, Bosch U, Tscherne H. The technique of packing for control of hemorrhage in complex pelvic fractures. Int Orthop. 1994;9(4):267-70.

12 Higgins JP, Altman DG. Chapter 8: Assessing risk of bias in included studies. In: Higgins JP, Green S, editors. Cochrane handbook for systematic reviews of interventions. Version 5.0.1 [updated September 2008]. Available at: http://hiv.cochrane. org/sites/hiv.cochrane.org/files/uploads/Ch08_Bias.pdf. (Accessed 01.02.17)

13 Wells GA, Shea B, O'Connell D, Peterson J, Welch V, Losos $M$, et al. The Newcastle-Ottawa Scale (NOS) for assessing the quality of nonrandomised studies in meta-analyses. Available at: http://www.ohri.ca/programs/clinical_epidemiology/ oxford. asp. (Accessed 01.02.17).

14 Tai D, Li W, Lee K, Cheng M, Lee K, Tang L, et al. Retroperitoneal pelvic packing in the management of hemodynamically unstable pelvic fractures: a level I trauma center experience. J Trauma. 2011;71(4):E79-86.

15 Osborn P, Smith W, Moore E, Cothren C, Morgan S, Williams A, et al. Direct retroperitoneal pelvic packing versus pelvic angiography: A comparison of two management protocols for haemodynamically unstable pelvic fractures. Injury. 2009; 40(1):54-60.

16 Li Q, Dong J, Yang Y, Wang G, Wang Y, Liu P, et al. Retroperitoneal packing or angioembolization for haemorrhage control of pelvic fractures--Quasi-randomized clinical trial of 56 haemodynamically unstable patients with Injury Severity Score $\geq 33$. Injury. 2016;47(2):395-401.
17 Hauschild O, Aghayev E, von Heyden J, Strohm PC, Culemann U, Pohlemann T, et al. Angioembolization for pelvic hemorrhage control: results from the German pelvic injury register. J Trauma Acute Care Surg. 2012;73(3):679-84.

18 Katsura M, Yamazaki S, Fukuma S, Matsushima K, Yamashiro $\mathrm{T}$, Fukuhara S. Comparison between laparotomy first versus angiographic embolization first in patients with pelvic fracture and hemoperitoneum: a nationwide observational study from the Japan Trauma Data Bank. Scand J Trauma Resusc Emerg Med. 2013;21:82.

19 Fang J, Shih L, Wong Y, Lin B, Hsu Y. Angioembolization and laparotomy for patients with concomitant pelvic arterial hemorrhage and blunt abdominal trauma. Langenbecks Arch Surg. 2011;396(2):243-50.

20 Tötterman A, Madsen JE, Skaga NO, Røise O. Extraperitoneal pelvic packing: a salvage procedure to control massive traumatic pelvic hemorrhage. J Trauma. 2007;62(4):843-52.

21 Ertel W, Keel M, Eid K, Platz A, Trentz O. Control of severe hemorrhage using C-clamp and pelvic packing in multiply injured patients with pelvic ring disruption. J Orthop Trauma. 2001;15(7):468-74.

22 Coccolini F, Stahelet P, Montori G, Biffl W, Horer TM, Catena F, et al. Pelvic trauma: WSES classification and guidelines. World J Emerg Surg. 2017;12:5.

23 Escobar GA, Cheng AM, Moore EE, Johnson JL, Tannahill C, Baker HV, et al. Stored packed red blood cell transfusion up-regulates inflammatory gene expression in circulating leukocytes. Ann Surg. 2007;246(1):129-34.

24 Aiboshi J, Moore EE, Ciesla DJ, Silliman CC. Blood transfusion and the two-insult model of post-injury multiple organ failure. Shock. 2001;15(4):302-6.

25 Miller PR, Moore PS, Mansell E, Meredith JW, Chang MC. External fixation or arteriogram in bleeding pelvic fracture: initial therapy guided by markers of arterial hemorrhage. J Trauma. 2003;54(3):437-43.

26 El-Haj M, Bloom A, Mosheiff R, Liebergall M, Weil YA. Outcome of angiographic embolisation for unstable pelvic ring injuries: factors predicting success. Injury. 2013;44(12):17505.

27 Burlew CC, Moore EE, Smith WR, Johnson JL, Biffl WL, Barnett CC, et al. Preperitoneal pelvic packing/external fixation with secondary angioembolization: optimal care for lifethreatening hemorrhage from unstable pelvic fractures. J Am Coll Surg. 2011;212(4):628-35; discussion 635-7.

Received June 15, 2017 Accepted after revision December 20, 2017 